\title{
DELEUZE ET BOULEZ
}

Pierre Montebello ${ }^{1}$

Resumé: Quelle est la place de Boulez dans la conception de la musique que se fait Deleuze? Il nous semble que c'est de montrer que le temps et l'espace sont musicaux, l'objet d'une double perception musicale. Ce n'est la musique qui est dans l'espace (partition) et le temps (exécution), mais l'espace et le temps qui relèvent d'une forme de musicalité. La musique est une expérience nostalgique de la spatialisation du temps (coexistence du passé/présent) et une expérience cosmologique de la synchronisation de l'espace (connexion des temps divergents du natal et du cosmos). Ecouter de la musique est un double déracinement de l'ici et maintenant, mais à travers les forces vives qui traversent le corps, voix célestes dans la martèlement des rythmes du corps, fuites cosmiques qui débordent l'enracinement du corps en son lieu natal.

Mots-clés: musique, temps, espace, perception musicale

Resumo: Qual é o lugar de Boulez na concepção da música de Deleuze? Parece-nos que é de mostrar que o tempo e o espaço são musicais, o objeto de uma dupla percepção musical. Não é a música que está no espaço (partitura) e no tempo (execução), mas o espaço e o tempo que se sobressaem de uma forma de musicalidade. A música é uma experiência nostálgica da espacialização do tempo (coexistência do passado/ presente) e uma experiência cosmológica da sincronização do espaço (conexão de tempos divergentes do nato e do cosmos). Escutar música é um duplo desenraizamento do aqui e agora, mas por meio de forças vivas que atravessam o corpo, vozes celestes no martelar dos ritmos dos corpos, fugas cósmicas que ultrapassam o enraizamento do corpo em seu lugar natal.

Palavras-chave: música, tempo, espaço, percepção musical

\begin{abstract}
Where does Boulez stand within Deleuze's music conception? It seems to us that Boulez is there to show us that both time and space are musical. It is not that music is inside the space (musical score) or inside the time (musical execution), but instead the space and the time are the ones which come out highlighted in some kind of musicality. Not only is music a nostalgic experience which positions time within space (the coexistence of past and present), but it is also a cosmological experience which synchronises space itself (the connection between two diverging times: the cosmological and the native). Listening to music is like uprooting both the "here" and the "now" at the same time, by using powers which cross through our bodies, by using celestial voices which come from the hammering in our bodies'rhythm, and by using cosmic escapes which overcomes the way our bodies root themselves in their own native place.
\end{abstract}

Keywords: music, time, space, musical perception

1 Maître de conférences à l'Université de Toulouse le Mirail. 
Foucault trace un beau portrait de Boulez:

Boulez n'a jamais admis l'idée que toute pensée, dans la pratique de l'art, serait de trop si elle n'était la réflexion sur les règles d'une technique et sur leur jeu propre. Aussi n'aimait-il guère Valéry. De la pensée, il attendait justement qu'elle lui permette sans cesse de faire autre chose que ce qu'il faisait. Il lui demandait d'ouvrir, dans le jeu si réglé, si réfléchi qu'il jouait, un nouvel espace libre. On entendait les uns le taxer de gratuité technique ; les autres d'excès de théorie. Mais l'essentiel pour lui était là : penser la pratique au plus près de ses nécessités internes sans se plier, comme de souveraines exigences, à aucune d'elles. Quel est donc le rôle de la pensée dans ce qu'on fait si elle ne doit être ni simple savoir-faire ni pure théorie ? Boulez le montrait : donner la force de rompre les règles dans l'acte qui les fait jouer. ${ }^{2}$

Laissons-nous porter par ce portrait. On ne va pas parler tout de suite des concepts que Deleuze emprunte à Boulez, « lisse », « strié, » « coupure »... mais de la transformation pratique opérée dans la musique par Boulez. Boulez se place clairement dans le camp d'une autonomie de la musique, dans la perspective de la création d'un « nouvel espace libre » musical, dans la direction d'une pensée qui opère par mutations pratiques.

Il revendique d'abord pour la musique un état d'autonomie. Agir en musique, c'est se réapproprier ce qu'elle fait. Avec Boulez, la révolution musicale prend la forme d'une autoréférence, d'une indépendance du langage musicale à l'égard de tout autre langage, et d'une nouvelle pratique. Boulez épouse finalement la version du formalisme qui est le grand récit dominant des arts contemporains picturaux et musicaux : l'art doit atteindre à propre autonomie. Dans les arts picturaux, on sait que ce récit remonte à Kant, l'autonomie de l'art pictural revient à se détacher de tout sens moral, politique, théologique. Pour la musique cela signifie que la musique n'a pas besoin d'un langage extérieur, elle est son propre langage. Et, la musique doit aussi acquérir son autonomie face aux pratiques musicales dominantes que sont les productions commerciales. Les ennemis de l'autonomie musicale sont le bruit informe et la chanson formatée. Bien sûr, on peut réintroduire le bruit en musique si on lui donne sa propre consistance musicale comme la musique spectrale ou la musique futuriste, on peut introduire la chanson bohème ou viennoise si on lui donne sa propre consistance musicale (comme chez Bartok, Schoenberg ou Mahler). L'important est que l'art atteigne son plan d'autonomie et de positivité où les matériaux consistent sans ajout.

Or, cette positivité a un nom chez Boulez : l'art est positif quand il découvre ce qui

2 Michel Foucault, "Pierre Boulez. L'écran traversé ", in Dits et écrits II, 1976-1988, Gallimard, "Quarto ", 2001, p.1041. 
le structure. La musique ne crée son plan de consistance que lorsqu'elle réduit son contenu et sa forme à une structure autonome. C'est en quelque sorte le structuralisme de Lévi-Strauss que Boulez introduit dans la musique. La musique n'a pas d'abord des sons et ensuite des formes, elle articule des structures locales mises en réseaux. A quelque niveau qu'on se place, la musique présente une matière/forme sonore où tout y est composé, où rien n'est donné. Le sérialisme découle de ces principes, de ce structuralisme. Le sérialisme en tant que mouvement musical créée un langage autonome à une micro-échelle sonore, cellulaire, moléculaire, qui ne découle d'aucune forme préétablie, d'aucune norme, d'aucune valeur dominante. Il adopte ainsi la position de Lévi-Strauss qui appelle forme «la mise en structure des structures locales, en quoi consiste le contenu ".3

Penser la musique paraît en 1963. Deleuze écrit lui son texte «A quoi reconnaît-on le structuralisme en 1967. Contenons-nous d'y reprendre trois formules de Deleuze qui auraient aisément pu être adoptées par Boulez s'il avait pu avoir connaissance du texte : 1) Toute structure est «topologique et relationnelle», elle consiste dans la position de ces éléments et leur mise en relation ; 2) «Toute structure est une micro-structure», définie par des rapports différentiels entre éléments; 3 ) «Toute structure est sérielle et multi-sérielle». Chez Boulez, la musique est topologique, relationnelle, micro-logique et multi-sérielle car elle ne se limite pas à l'indépendance des 12 sons de la gamme chromatique tempérée, à savoir à la hauteur du son, mais s'intéresse aux durées, timbres, intensités des sons en tant que séries. On peut au final concevoir la musique sérielle comme le symbole d'un univers non-répétitif, en perpétuelle transformation et expansion, «un mode de pensée polyvalent » qui s'élargit à la structure même de l'œuvre qu'elle engendre, une musique à la fois imprévisible, sans direction, en variation continue.

Boulez dit rejoindre le structuralisme de Lévi-Strauss. Mais, en retour, Lévi-Strauss marque sa désapprobation de la musique sérielle, en raison de l'écart abyssal entre perception et compréhension. L'œuvre sérielle est quasi imperceptible. A la manière des œuvres visuelles de Reinhardt impossibles à voir, elle est difficile à écouter, presqu'inécoutable, elle se soustrait à l'acte même de l'audition parce qu'elle est fluide, dissonante, abstraite, sans repères auditifs. Elle est une idée plutôt qu'une perception subjective. Les œuvres de Boulez sont écrites et destinées à êtres réécrites sans fin. Inachevées par essence, leur élaboration se substitue à leur exécution. La disparation de la perception au profit d'une conception musicale désincarnée, imperceptible, métallique, c'est précisément ce que Lévi-Strauss n'accepte pas.

Il se pourrait que la musique sérielle relève d'un univers où la musique n'entraînerait pas l'auditeur dans sa trajectoire, mais l'éloignerait de lui. En vain il s'évertuerait à la rejoindre : chaque jour elle lui ap-

3 Penser la musique aujourd'hui, Denoël, 1963, p. 31. 
paraitrait plus lointaine et insaisissable. Trop distante, bientôt, pour l'émouvoir, seule son idée resterait encore accessible, avant qu'elle ne finisse par se perdre sous la voûte nocturne du silence, où les hommes ne la reconnaîtront qu'à de brèves et fuyantes scintillations4.

Deleuze lui prend le parti opposé : il s'en inspire pour autre chose comme il le dit dans sa conférence à l'IRCAM. A quoi correspond la distinction du « lisse » et du « strié » que Deleuze emprunte à Boulez ? A deux manières de nous connecter au monde. Rappelons que cette grande distinction est d'abord spatiale. Elle institue une répartition spatiale dans la musique que Deleuze va appliquer au politique (nomades et sédentaires) et au cosmologique (le mouvant et le statique). La grande thèse de Boulez est que la musique joue sur cinq dimensions : les hauteurs, les durées, les intensités, les timbres, et l'espace. L'espace est sa cinquième dimension, la plus mentale en un sens, parce que la plus imperceptible.

La musique crée donc des espaces perceptifs, elle s'apparente à une architecture en sons où le topologique n'a pas moins de sens que le chronologique. Les espaces engendrés sont de deux sortes, lisses ou striées. Soit qualitatifs, soit statistiques. Un espace sonore peut être est strié par des qualités tonales, ou il peut être lissé par un chaos statistique. Mais comment Boulez en vient-il à un tel problème ? Il lit Poincaré et s'intéresse à la question de la coupure du continu, et aux espaces qu'une telle coupure engendre (ensemble des nombres rationnels, ensemble des nombres irrationnels). Et il transpose ce problème du continu à la musique. Mais, allons à l'essentiel : le lisse désigne un type d'espace sonore avec ses directions, le strié un type d'espace sonore avec des dimensions qualitatives. Pourquoi cette distinction intéresse-t-elle Deleuze ? Pourquoi ce souci du spatial dans le temps musical?

Dans sa conférence à l'IRCAM, Deleuze met l'accent sur les espaces sonores non subjectifs, « le dialogue de la mer et du vent », « les intensités silencieuses », « les rythmes de toute nature », « le temps à l'état pur » ... L'espace sonore se peuple de formes non humaines indépendantes de la « hiérarchie matière-vie-esprit 》 et de ses cadences ou mesures propres. L'homme ne bat plus la mesure des choses. Deleuze trouve dans la musique de Boulez et le sérialisme ce qu'il va très précisément rechercher dans les autres arts, picturaux ou cinématographiques : le montée de la terre, du moléculaire, et du cosmique dans la perception humaine. Tout un géomorphisme avec ses paysages. On se souvient des trois moments de la ritournelle, sa manière de composer avec les « Forces du chaos ; forces, terrestres, forces cosmiques ». C'est partout la même ritournelle chez Deleuze. Partout on entend la même musique : il arrive un moment où l'art se connecte à des forces qui le déterritorialisent, le déracinent de la scansion des milieux, des pers- 
pectives auto-centrées : ça arrive au cinéma avec Dovjenko où le chant de « La terre » passe "dans toutes les chansons de l'homme » pour former un grand chant révolutionnaire cinématographique ; ça arrive dans la peinture quand le chant de l'univers passe des paysages non humains pour créer un univers sans homme comme dans la peinture de montagne sainte Victoire par Cézanne ; ça arrive en littérature quand elle fait sait nous faire entendre les chants de la steppe, de la forêt, de l'immensité liquide ; ça arrive dans la premier mouvement de la troisième symphonie de Mahler où le chant de la terre déborde tous les territoires, oiseaux, forêts, couleurs...

Partout, dans tous les livres, une même petite musique, une même ritournelle deleuzienne. La musique est l'écoute du dehors inhumain, la connexion avec un plan de nature première que les forces de l'art composent:

D'une certaine manière, la musique classique européenne pourrait se définir dans le rapport d'un matériel auditif brut et d'une forme sonore qui sélectionnait, prélevait sur ce matériel.(...). Au couple matériel brut-formes sonores, se substitue un tout autre couplage matériau sonore élaboré-forces imperceptibles que le matériau va rendre audibles, perceptibles. Peut-être un des premiers cas les plus frappants serait dans le dialogue du vent et de la mer de Debussy. Dans le cycle proposé par Boulez, ce serait la pièce II, modes de valeurs et d'intensité, et la pièce IV, Eclat.

La musique de Boulez ou de Debussy nous ferait donc entendre l'imperceptible Dehors inhumain, ces « forces d'une autre nature », non sonores en elles-mêmes, c'est en ce sens que la musique aurait un rapport avec les autres arts. Reprenons l'exemple de la mer : il ne s'agit pas de copier le bruit de la mer, mais de créer une mélodie qui devienne paysage maritime en prenant en contre-point les aspects de ce paysage virtuel qu'est la mer. Ce paysage mélodique manifeste par la musique les forces non sonores de la mer, son immensité, son ressac éternel, sa puissance terrifiante ... Voilà ce que la musique doit réussir à nous faire entendre, qui n'est certes pas le bruit de la mer ! Or, c'est bien aussi, mais d'une autre façon, ce que la littérature de Melville prend pour objet dans Moby Dick, l'immensité, le sublime, le trop grand pour être pensé, l'objet absolu. Dans sa quête de l'objet immense (Moby Dick), comment Achab ne côtoierait-il pas le néant? Qui peut assurer que celui qui franchit le mur de la perception ne se retrouvera pas en plein néant plutôt que dans l'absolu ? Et, c'est bien alors que la littérature de Melville rejoint la musique, quand il nous donne à entendre la langue de la Baleine, déterritorialisée, outlandish, « langue originaire inhumaine ou surhumaine ». Voilà que la littérature est elle-même traversée par des composantes musicales lorsqu'elle découvre son propre Dehors, « silence ou musique », ce que Deleuze appelle « l'au-delà musical et céleste du langage tout en- 
tier ». Au-delà musical d'une Nature première qui féconde la nature seconde et humaine. Et, ce n'est pas le problème de l'art humain, c'est le problème de tout art. L'oiseau musicien n'est pas si loin de Melville, parce qu'il connecte territoire et langage déterritorialisé. Son chant ne consiste pas en signes territoriaux, il compose un paysage mélodique qui a pour contre point ses affects intérieurs et ses milieux extérieurs rendant sonore « le soleil, la joie ou la tristesse ». Je disais que la question de l'art est traversée par la même ritournelle chez Deleuze, partout la même ritournelle se fait entendre : la musique n'est pas seulement l'affaire des musiciens :

Un matériau sonore très complexe est chargé de rendre appréciables et perceptibles des forces d'une autre nature, durée, temps, intensité, silences, qui ne sont pas sonores en elles-mêmes. (...) la musique a rendu de plus en plus audibles ce qui la travaillait de tout temps, des forces non sonores comme le Temps, l'organisation du temps, les intensités silencieuses, les rythmes de toute nature. Et c'est là que les non musiciens peuvent, malgré leur incompétence, se rencontrer le plus aisément avec les musiciens. La musique n'est pas seulement l'affaire des musiciens, dans la mesure où elle rend sonore des forces qui ne le sont pas, et qui peuvent être plus ou moins révolutionnaires, plus ou moins conformistes, par exemple, l'organisation du temps. (Conférence IRCAM).

Reposons la question : qu'est-ce que Deleuze a trouvé chez Boulez qui le concernait, c'est-à-dire qui entrait dans sa ritournelle philosophique ? Deleuze le dit ici : Boulez l'a aidé à répondre à ses grandes questions sur le temps et l'espace. Il y a en musique un rapport évident entre la partition spatiale et son exécution temporelle. Mais ce n'est pas de cela dont nous voulons parler. Ce rapport est arbitraire encore. Il faudrait se demander si le temps et l'espace ne sont pas musicaux dans leur essence. Il y aurait un rapport profond, décisif, essentiel entre musique, espace et temps. Au fond, la la musique est l'art qui met le plus complètement en forme le dédoublement du temps et de l'espace dans nos expériences, mais seulement parce que le temps et l'espace sont déjà musicaux. Qu'est-ce que le temps si ce n'est une ritournelle musicale, un da capo, un dédoublement passé/ présent? Qu'est-ce que l'espace chez Boulez si ce n'est avant tout une affaire musicale, une structuration musicale, espace lisse ou strié ? C'est en ce sens que les autres arts sont concernés par la musique, que tous les arts sont musicaux.

Le privilège de la musique est en effet d'exprimer à la fois le dédoublement du temps entre passé et présent et la division de l'espace entre la Terre et le déterritorialisé. A toutes les époques, la musique est confrontée à ce problème. Nous n'existons pas sans être déchirés par le temps et l'espace, sans être temporalisés par le temps et spatialisé par l'espace. Ecoutez de la musique, c'est subir et sentir à même le corps cette double torsion, 
la torsion du temps en nous et de l'espace en nous. Nous sommes comme des bandes de Moebius intérieurement. C'est cela que Deleuze appelle cristal de temps, le passage réciproque du passé et du présent l'un dans l'autre. On pourrait aussi bien parler d'un temporal d'espace, qui serait la synchronisation de la terre et du déterritorialisé, leur passage l'un dans l'autre. Toujours un pied dans un territoire, et un autre à suivre une ligne de déterritorialisation, comme dans le romantisme tiraillé entre le natal et une ligne de fuite cosmique. Dans la musique, le son nous pousse, nous traverse, nous entraîne, il exprime la bifurcation le temps, par la juxtaposition du passé et du présent, et il exprime la division temporelle de l'espace en synchronisant le rythme terrestre et la mélodie céleste. Il est étrange de voir combien chez Deleuze, le temps est toujours ramené à des juxtapositions d'espace, tandis que l'espace se donne toujours dans des synchronisations du temps, rythme et mélodie. Le son est simultanément espace et temps, rapport à la Terre/Comos et au Passé. Par lui passent la mort comme passé pur et la déterritorialisation comme abandon du natal:

C'est une ligne phylogénique, un phylum machinique qui passe par le son, et en fait une pointe de déterritorialisation. Et cela ne va pas sans de grandes ambiguïtés : le son nous envahit, nous pousse, nous entraîne, nous traverse. Il quitte la terre mais aussi bien pour nous faire tomber dans un trou noir que pour nous ouvrir à un cosmos. Il nous donne l'envie de mourir. ${ }^{5}$

Je disais : il y a une ritournelle philosophique chez Deleuze, qui explique que la musique est le langage de tous les non musiciens, et qu'on la retrouve au cinéma, en littérature, en peinture... On la retrouve dans tous les arts parce que tous sont concernés par l'épreuve du temps et de l'espace et que ces épreuves sont musicales en leur essence. Le temps est une ritournelle non moins que l'espace.

Que le temps et l'espace soient en leur essence des formes musicales, c'est ce que Deleuze en vient à soutenir dans ses deux livres sur le cinéma. Il attribue cet apport, le plus spécifique, à Guattari : «Félix Guattari avait raison de définir le cristal de temps comme étant par excellence une 'ritournelle'». C'est donc le temps lui-même qui est une ritournelle, musical en son fond. A première vue, tout cela semble très abstrait : la ritournelle musicale exprimerait un « cristal de temps », le cycle du passé et du présent; le temps-ritournelle lui-même. Mais, en réalité cette proposition est assez concrète. L'on perçoit toujours dans la musique deux moments du temps. Un moment terrestre, le martèlement de pas sur le sol, la précipitation du présent, comme le « galop » d'un cheval, rythme effréné. Et puis le passé qui se conserve, la mélodie céleste qui nous arrache à la 
terre, qui nous élève, qui éternise « un commencement de monde », soustrait au temps qui passe, fragment intemporel du temps. La musique est cette dualité, galop et mélodie, rythmes et chant, percussions et voix. C'est ce qui fait que la musique est si émouvante. Le corps est traversé par le son, il vibre dans toutes ses attaches terrestres, il résonne dans le temps d'ici, au sein d'une immense déperdition entropique, d'une chute des présents, tandis que monte le chant nostalgique et mélancolique qui soustrait le corps au temps, qui le hisse en contemporain d'un passé à jamais révolu, en fait le témoin d'une grâce légère, le siège d'une éternité soudaine. Voilà ce que Deleuze dit de la musique de Nino Rota dans Prova d'orchestra de Fellini : «On entend d'abord le pur galop des violons, mais se lève insensiblement une ritournelle jusqu'à ce que les deux s'insinuent l'un dans l'autre de plus en plus intimement, s'étreignant comme des lutteurs, perdus-sauvés, perdus-sauvés... ». La musique comme expérience de la grâce. Sauvés-perdus.

Ainsi s'explique que la musique soit une expérience fondamentalement à part. C'est en découvrant Boulez que Deleuze s'aperçoit que le temps et l'espace ont cette étoffe musicale. Que le temps et l'espace vibrent en nous, et que les sons révèlent ces forces. Nous voyons bien que dans l'espace-temps lisse et que dans l'espace-temps strié, pour reprendre les concepts de Boulez utilisés par Deleuze, nous avons un rapport à la terre et au déterritorialisé, nous sommes directement en contact avec des forces étrangères, solidaires de ces forces, pénétrés par ces forces. C'est ce qui fait la «supériorité de la musique» comme le dit Logique de la sensation, cette expérience de la torsion des forces en nous. Le choc de la peinture est physique, il concerne la vision, la perception de la matière dont les corps sont faits. Certes la peinture peut être une expérience hystérique quand le réel est si violent que sa présence nous dévaste. Les figures convulsionnés de Bacon et le cinéma de Voyants qu'est le néo-réalisme italien en attestent : on peut être suffoqué par le réel qui fait du corps entier un espace de vision : l'enfant de Allemagne année zéro de Rossellini meurt littéralement de ce qu'il voit. Mais, la musique est une autre expérience. Ce n'est pas une expérience de la vison ou de Voyant, c'est une expérience nostalgique de la spatialisation du temps (coexistence du passé/présent) et une expérience cosmologique de la synchronisation de l'espace (connexion des temps divergents du natal et du cosmos). Ecoutez de la musique est un double déracinement de l'ici et maintenant, mais à travers des forces vives qui traversent le corps, voix célestes dans la martèlement des rythmes du corps, fuites cosmiques dans l'enracinement du corps en son lieu natal. C'est dans ce contexte que les distinctions que Deleuze et Guattari empruntent à Boulez ont une signification précise. Le temps lisse et le temps strié désignent la manière dont le continuum du temps est rompu en musique pour donner un temps pulsé ou un temps sans mesure. Ce ne sont pas du tout les mêmes temps et les mêmes expériences. Le temps pulsé parle à nos oreilles. La musique pulsée est rythmée par des repères auditifs, elle est à notre mesure. Quelle se définisse dans la musique classique par le tempérament (que celui-ci soit méso- 
tonique, égal, ou bien inégal, n'est pas sans signification) : les sons dans un temps pulsé nous affectent naturellement parce qu'ils sont rythmés pour nos oreilles. La relation entre tonalité et tempérament était très codifié comme le montre les règles de composition écrites par Charpentier au XVIIème siècle : «Do majeur, gai et Guerrier, do mineur obscur et triste, ré mineur, grave et dévot, ré majeur, joyeux et très guerrier, mi bémol majeur, cruel et dur, mi bémol mineur, horrible affreux, (...) si mineur, solitaire et mélancolique.» Dans un tel système où la note a une valeur dans l'axe horizontal de la mélodie et dans l'axe vertical de l'harmonie, il est besoin à la fois de mémoire et de culture d'un style pour que la musique nous parle. Nous avons bien affaire à une représentation musicale, un espace sonore dominée par des structures et des formes en relation avec notre tempérament.

Rien de tel dans le temps amorphe où les repères auditifs ont disparu. L'oreille ne peut plus se repérer, elle est plongée dans un espace nomade parcouru de flux sonores qui attaquent de plein fouet le système nerveux sans plus être cryptés par l'oreille. Deleuze trouve dans Boulez un espace sonore nomade, un continuum qui est une variation infinie sans repères où Daniel Charles voit la possibilité d'une «musique de l'oubli». Impossible en effet de se remémorer dans ce flux sonore : maintenant les forces sonores saturent l'espace sans intervention psychologique. Essentiellement oubliée, il n'y a qu'ainsi que la musique se fait entendre. Le diagramme musical abolit les représentations, les mémoires, les repères. Pascale Criton, musicienne contemporaine, a suivi les cours de Deleuze et participé à de nombreuses discussions sur la musique avec lui avant l'écriture de Mille Plateaux. Elle raconte : «La notion de diagramme et de production transversale du modèle musical, celui des espaces lisses et des espaces striés en particulier (...), fit l'objet de lectures et de réflexions. Nous eûmes de nombreuses réflexions sur les séries harmoniques, les partiels et composantes du son, en particulier au cours de l'étude de Leibniz, dont Deleuze recommandait fortement la lecture aux musiciens. D'autres fois, nous développions des recherches à propos des affects et de l'inflexion vocale dans la musique baroque, ou encore des signaux et comportements animaliers et des formes qui pouvaient en découler, de la caccia aux rondo et ritornelli. C'est ainsi que certaines notions sont devenues déterminantes dans Mille Plateaux. ${ }^{6}$

Pascale Criton pose alors la question essentielle:

Quelle est l'affaire de la musique, quel est le contenu indissociable de l'expression sonore? Guattari et Deleuze font de la musique le lieu privilégié d'un processus transversal de variation. Lieu d'échange ten$\mathrm{du}$ entre les forces territorialisantes de la ritournelle et la composition d'une ligne de variation proprement musicale. La ritournelle fait point de préhension, territoire, pli sécuritaire, avec le risque d'un retour mé-

6 Pascale Criton, L'invitation, approches et portraits, Herman, 2005, p. 60. 
lancolique au natal, mais c'est essentiellement une ligne potentielle dont les points peuvent se redistribuer, se mettre en mouvement: distribution polyphonique, variations mélodiques, variations de timbres, de vitesses, de dynamiques, de densité d'orchestration...

Et de citer Deleuze: «La musique est l'opération active, créatrice, qui consiste à déterritorialiser la ritournelle».

Pascale Criton a, dans sa composition musicale, une certaine fidélité envers Deleuze qui peut nous intéresser ici. Elle a suivi les cours de Deleuze, mais elle a aussi suivi l'enseignement du maître de la musique micro-tonale Ivan Wyschnegradsky : ce compositeur remarquable écrit dans des tiers, des quarts, ou des sixièmes de ton, soit des micro-intervalles de temps qui donnent l'impression d'un glissement continu du son, d'une profonde étrangeté sonore, avec une consistance sonore propre. Or, le problème là encore est celui d'une spatialisation du son, comme chez Boulez. La continuité sonore micro-tonale engendre une cartographie nouvelle du paysage sonore. Wyschnegradsky défend, avec «La loi de pansonorité», l'idée que les espaces sonores engendrés par les micro-intervalles révèlent un «champ vibratoire infini». Dans cette lignée, Pascale Criton élabore une musique faite de continuités de timbre quasi imperceptibles, de micro-variations. Dans ces morceaux, la continuité sonore semble être une perpétuelle différenciation d'une même base. Est-ce un hasard si ses morceaux s'intitulent Territoires imperceptibles (lettres M-N), Passage des heures, La ritournelle et le galop?

Tout est bien ici l'affaire de cartographies spatiales. Mais, on pourrait se demander aussi si un tel espacement musical ne serait pas plus en phase avec le minimalisme qu'avec le sérialisme ou le micro-tonal. Le minimalisme choisit fermement de réinstaurer la tonalité, et même une hypertonalité contre le sérialisme trop marqué par des enjeux de pouvoir théorique ou politique. C'est une autre manière de se rapporter à la musique, plus libre, qui apparaît dans les années 60 et 70, avec Steve Reich, Philipp Glass, ou Arvo

ärt. Tel est l'avis de Joachim Dupuis dans son article, 《 Variations, métamorphoses et cristal- à propos de la rencontre entre musique expérimentale et minimalisme $"{ }^{7}{ }^{7} \mathrm{On}$ a cru déceler dans le minimalisme une régression anté-boulézienne ou un sorte de restauration post-révolutionnaire de la tonalité, alors qu'il faudrait plutôt y voir une manière de composer avec la force nomade du Dehors. Le corps est pris dans une dynamique, des intensités qui passe par le retour à la voix, aux chants tribaux voire aux rythmes religieux (Tehilim de Reich, Te Deum de Pärt). Joachim Dupuis n'hésite pas à dire que c'est « une musique éminemment végétale, animale, minérale au sens où Deleuze parlait de deve-

7 L'article de Joachim Dupuis est disponible en ligne: http://www.musicologie.org/publirem/dupuis_01. html 
nir-animal, végétal ou minéral » : «Les métamorphoses de la musique semblent suivre des lignes actives et parcourir le monde. Devenir-minéral (The desert music de Reich, ou la symphonie $n^{\circ} 3$ de Glass). Devenir-historique des USA (avec Nixon in China). Ou encore Devenir-pelliculaire (The photographer de Glass). En ce sens, cette musique est une musique mineure ».

Il n'est pas sûr que Deleuze ait eu un goût prononcé pour la musique sérielle, et peut être pas davantage pour le minimalisme. En tout cas, dans l'abécédaire, ses penchants musicaux ne vont pas directement à cette période. Mais, peu importe le choix du sérialisme ou du minimalisme, ou même de la chanson française qu'il semble préférer, avec Boulez c'est bien la spatialisation du temps et la temporalisation de l'espace qui intéresse Deleuze. Le rapport aux espaces, milieux, territoires, cosmos, ne doit pas faire oublier que la découverte de la puissance diagrammatique de la musique réside dans sa capacité à engendrer des espaces et des temps décentrés, non psychologiques, non mémoriels. Les forces-matériaux sonores, en percutant notre corps, engendrent un dédoublement du temps et une division de l'espace, produisent des affects nostalgiques et cosmologiques qui sont la seule promesse d'un devenir possible ce corps. Expérience unique d'une grâce instantanée : sauvés/perdus, perdus/sauvés.

\section{Referências Bibliográficas:}

FOUCAULT, M. “Pierre Boulez. L'écran traversé ”, in Dits et écrits II, 1976-1988, Paris, Gallimard, “Quarto ”, 2001

BOULEZ, P. Penser la musique aujourd'hui, Paris-Genève, Ed. Gonthier/ Denöel, 1963 LÉVI-STRAUSS, C. Le cru et le cuit, Paris, Plon, 1964.

DELEUZE, G. et GUATTARI, F. Mille Plateaux, Paris, Éditions de Minuit, 1980

CRITON, P. L'invitation, Approches et portraits, Paris, Hermann, 2005

DUPUIS, J. - article disponible en ligne: http://www.musicologie.org/publirem/dupuis_01.html 